\title{
Grape Seed Proanthocyanidin Extract Adjusts NeuN/GFAP in a Murine Model of Neonatal Hypoxic-ischemic Brain Injury
}

\author{
Li Luo ${ }^{1, \dagger}$, Xing Tu${ }^{1, \dagger}$, Xuexia Zhang ${ }^{2}$, Jing Liu', Yilin Liu ${ }^{3}$, Wenyan Zhao ${ }^{3}, K^{1}$ Niu $^{3}$, Junhua Yang ${ }^{1}$, \\ Guoying Li ${ }^{1, *}$, Mengxia Wang ${ }^{4}$, * \\ ${ }^{1}$ School of Biosciences \& Biopharmaceutics, Guangdong Pharmaceutical University, Guangzhou, People's Republic of China \\ ${ }^{2}$ Department of Anesthesiology, The Fifth Affiliated Hospital, Sun Yat-Sen University, Zhuhai, People's Republic of China \\ ${ }^{3}$ School of Clinical Medicine, The First Affiliated Hospital of Guangdong Pharmaceutical University, Guangzhou, People's Republic of \\ China \\ ${ }^{4}$ Intensive Care Unit, Guangdong No. 2 Provincial People's Hospital, Guangzhou, People's Republic of China
}

Email address:

gracemengxia@hotmail.com (Mengxia Wang), gzlgying820@sina.com (Guoying Li)

${ }^{*}$ Corresponding author

${ }^{\dagger}$ Li Luo and Xing Tu are co-first authors.

\section{To cite this article:}

Li Luo, Xing Tu, Xuexia Zhang, Jing Liu, Yilin Liu, Wenyan Zhao, Ke Niu, Junhua Yang, Guoying Li, Mengxia Wang. Grape Seed Proanthocyanidin Extract Adjusts NeuN/GFAP in a Murine Model of Neonatal Hypoxic-ischemic Brain Injury. American Journal of Pediatrics. Vol. 5, No. 2, 2019, pp. 51-55. doi: 10.11648/j.ajp.20190502.13

Received: March 20, 2019; Accepted: April 22, 2019; Published: May 10, 2019

\begin{abstract}
Objective: To investigate the neuroprotective effects of grape seed proanthocyanidin extract(GSPE) on a model of neonatal hypoxic ischemic encephalopathy (HIE), we investigated the changes in neuronal cells and astrocytes after pre-treatment with GSPE. Methods: Seven-day-old pups were randomly divided into sham, HI, and GSPE+HI groups. The HIE model was established using a modified Rice-Vannucci method. GSPE was injected intraperitoneally 20 min before surgery. The change in markers of neuronal cells and astrocytes (NeuN/GFAP) were detected by immunofluorescence and Western blot. Results: Compared with the sham group, the expression of NeuN in the HI group was significantly reduced, and the expression of NeuN was significantly increased after GSPE pre-treatment. The expression of GFAP was opposite to NeuN. Conclusion: Our study showed that GSPE pre-treatment significantly protected neurons and inhibited astrocyte over-proliferation. Therefore, we believe that GSPE is a potential drug for the treatment of HIE and can prevent brain damage caused by hypoxia and ischemia.
\end{abstract}

Keywords: Grape Seed Proanthocyanidin Extract, NeuN, GFAP, Neonatal Hypoxic-Ischemic Brain Injury

\section{Introduction}

Hypoxic-ischemic encephalopathy (HIE) is a brain injury caused by perinatal asphyxia. HIE often leads to severe brain damage and cognitive dysfunction [1-2]. According to the available data, $25 \%-30 \%$ of infants who survive HIE brain injury develop long-term severe sequelae [3], such as cerebral palsy, epilepsy, and even mental disorders [4-5]. This catastrophic disease not only significantly reduces the quality of life of patients, but also causes a heavy financial burden to families and countries.
Despite the increasing awareness of HIE, there is still a lack of positive and effective measures to intervene in this disease.

Grape seed proanthocyanidin extract (GSPE) is a polyphenolic compound [6-7] that is a common natural antioxidant [8]. GSPE treatment has shown good therapeutic effects in models of esophageal squamous cell carcinoma [9], hypertension [10], diabetes [11], and other diseases. Our previous results indicate that GSPE pre-treatment can significantly improve brain damage in a HIE murine model, and we have discussed the 
neuroprotective effect from the perspective of autophagy/apoptosis/behavioral cognition. Neurons and astrocytes are the two main types of nerve cells in the brain. Previous studies have shown that when HIE injury occurs, it often leads to irreversible death of neurons in the brains of HIE mice, and large accumulation of astrocytes on the injured side can often be observed [12]. To investigate the effect of GSPE pre-treatment on these two types of nerve cells, we utilized immunofluorescence and Western blot to explore the changes in neurons and astrocytes before/after GSPE administration to provide a theoretical basis for GSPE pre-treatment of hypoxic-ischemic brain injury in the future.

\section{Materials and Methods}

\subsection{Establishing a Model of Neonatal HIE}

We chose 7-day-old mice for the following experiments. Experimental mice were purchased from the Laboratory Animal Center of Sun Yat-sen University. These mice were randomly divided into the following three groups: sham; HI; and GSPE+HI. All procedures were approved by the Ethics Committee of Guangdong Pharmaceutical University and the guidelines of the Council on Animal Care were strictly followed. We established the HIE model by using the modified Rice-Vannucci method, which is widely accepted [13]. Briefly, 7-day-old pups were anesthetized with isoflurane and the common carotid artery (CCA) on the left side was ligated. Then, the mice were put back into the cage for $60 \mathrm{~min}$ to recover. Next, the mice were placed in a small square airtight space, which was continuously filled with low oxygen $(8 \%$ $\mathrm{O}_{2}+92 \% \mathrm{~N}_{2}$ ) for $2 \mathrm{~h}$. Sham controls underwent anesthesia and the left CCA was exposed as in the HI group, but without ligation or exposure to hypoxia.

\subsection{GSPE Administration}

Powdered GSPE (Meilun Biological, Dalian, China) was dissolved in PBS solution at a concentration of $1.25 \mathrm{mg} / \mathrm{ml}$. The GSPE+HI and HI groups were intraperitoneally injected with different substances at a dose of $30 \mathrm{mg} / \mathrm{kg} 20 \mathrm{~min}$ before surgery. The GSPE+HI group was injected with GSPE solution, and the HI group was injected with PBS solution (the same volume as the control group).

\subsection{Immunofluorescence Staining}

We obtained samples 3 days after surgery. Specifically, the mice were perfused transcardially and fixed with $4 \%$ paraformaldehyde. The extracted brain tissue was dehydrated in ethanol and xylene, then the brain tissue was made into paraffin blocks using an embedding machine. Then, the brain tissues were cut into 3-5 micron thick slices for the following experiments. We selected appropriate brain slices for immunofluorescence staining. After the brain slices were dewaxed with xylene and ethanol, the brain slices were placed in $0.01 \mathrm{M}$ citrate buffer, and antigen repair was performed under high pressure for $20 \mathrm{~min}$. We added goat serum to the brain slices for $1 \mathrm{~h}$, then incubated the brain slices with the following two antibodies in a $4{ }^{\circ} \mathrm{C}$ refrigerator overnight: anti-NeuN (dilution, 1:800; Sigma, USA); and anti-GFAP (dilution, 1:800; Abcam, UK). The next day we incubated the fluorescence secondary antibody in the dark room. After sealing the brain slices, we observed the brain slices under a fluorescence microscope.

\subsection{Western Blotting}

We extracted brain tissue proteins on the third day after surgery. We extracted ipsilateral brain tissue and added RIPA lysate and PMSF to the brain tissue. After full grinding of the brain tissue into fragments, the brain homogenate was centrifuged in a frozen centrifuge at $4^{\circ} \mathrm{C}$ for $15 \mathrm{~min}(12,000$ $\mathrm{rpm} / \mathrm{min}$ ). We extracted the supernatant for the following experiments. Quantitative analysis was performed with a BCA Protein Quantitative Kit (Beyotime Biotechnology, Shanghai, China). The protein was concentrated and separated on SDS-PAGE gels and transferred to PVDF membranes (Millipore, Schwalbach, Germany). We incubated the NeuN (dilution, 1:1500; Sigma, USA) and GFAP antibodies (dilution, 1:1500; Abcam, UK) with the obtained protein stripes. $\beta$-actin $(1: 2000)$ was used as an internal control protein. After the corresponding secondary antibodies were incubated, image J and SPSS 19.0 software were used to analyze the results.

\subsection{Data Analysis}

Data are presented as the mean \pm SEM. Three or more groups of data were analyzed by one-way ANOVA, followed by a Bonferroni test if more than two groups were involved. A $\mathrm{P}<0.05$ was considered statistically significant. All analyses were performed using SPSS 19.0 software and Graph Pad Prism 4.0.

\section{Results}

GSPE pre-treatment significantly increased the expression of NeuN protein and decreased the expression of GFAP protein.

To prove that GSPE pre-treatment increased the expression of NeuN and reduced the expression of GFAP, we performed immunofluorescence staining on brain slices (Figure 1A, 1B). Immunofluorescence staining of ipsilateral brain tissues showed that NeuN protein expression was decreased when HI injury occurred. After GSPE pre-treatment, the expression of NeuN was significantly increased. In contrast, compared with the HI group, GFAP expression was significantly decreased after GSPE pre-treatment. 


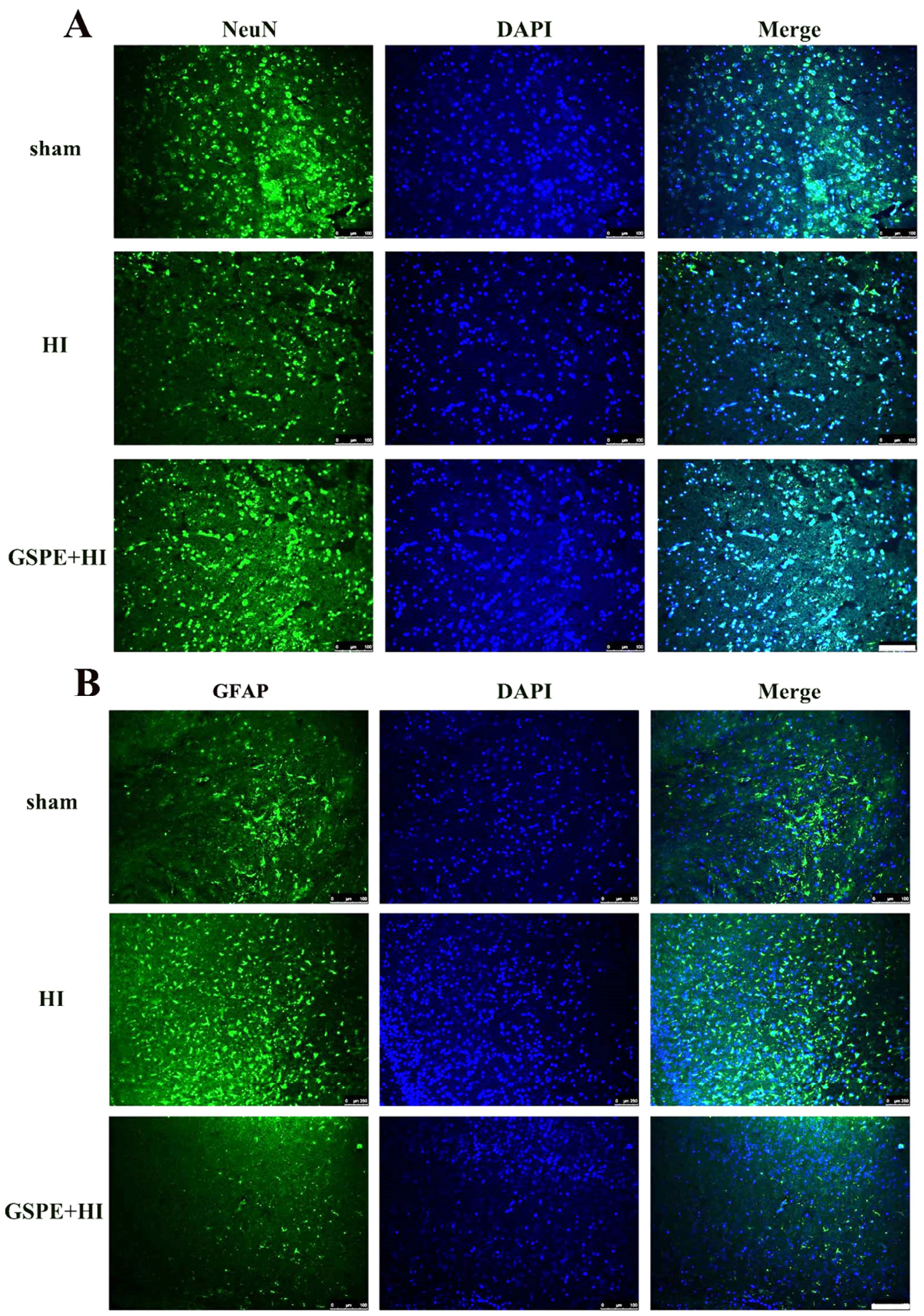

Figure 1. GSPE pre-treatment increased the expression of NeuN and decreased the expression of GFAP in the HIE model.

Representative immunofluorescence of NeuN and GFAP in ipsilateral brain tissues (A and B). The positive cells were stained green and the nuclei stained blue. Compared to the sham group, the expression of NeuN decreased in the HI group in the ipsilateral brain. In the GSPE+HI group, the expression of NeuN increased more than the HI group; the expression of GFAP was the opposite. Scale bar $=100 \mu \mathrm{m}$

As shown above, GSPE pre-treatment significantly increased the expression of NeuN protein and decreased the expression of GFAP protein. To further test the change in NeuN/GFAP, we quantitatively analyzed the two proteins 
with Western blot (Figure 2 B, C). The changes in NeuN and GFAP expression were consistent with the results of immunofluorescence. These data suggest from different

A

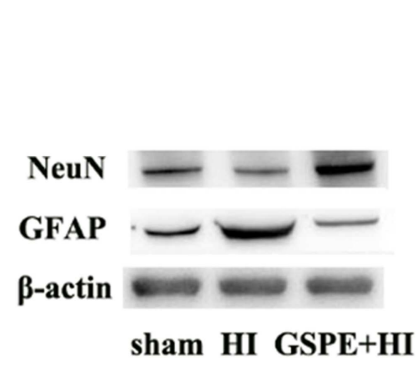

B

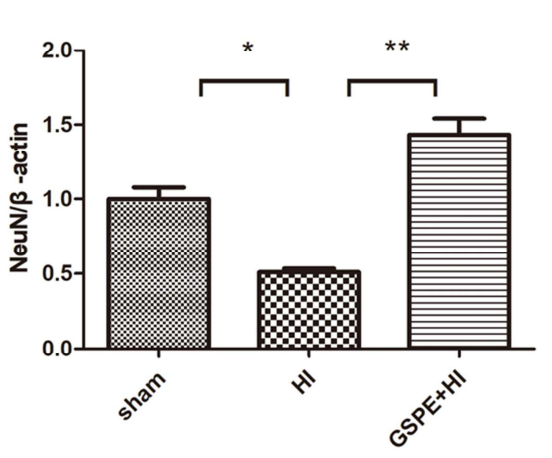

perspectives that GSPE pre-treatment can protect neurons and inhibit the excessive proliferation of astrocytes.
$\mathrm{C}$

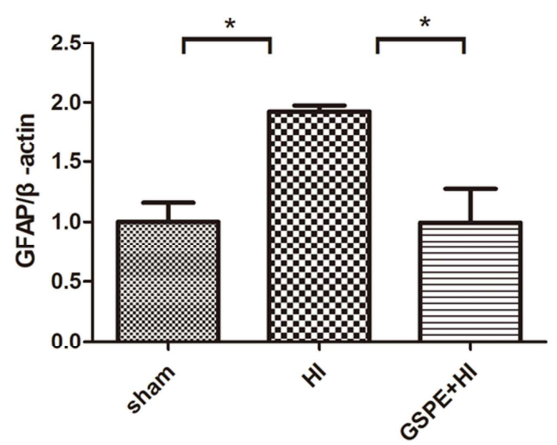

Figure 2. GSPE pre-treatment induced NeuN and reduced GFAP in the HIE model.

Representative Western blots (A) and quantification data (B) (C) of NeuN and GFAP are shown. In the HI group, the expression of NeuN was less than the sham group. After pre-treatment with GSPE, the expression of NeuN increased. The expression of GFAP was opposite to NeuN. All calculations were normalized to $\beta$-actin. $* \mathrm{P}<0.05, * * \mathrm{P}<0.01$. The difference was considered statistically significant by one-way ANOVA followed by the Bonferroni test.

\section{Conclusion}

In summary, we explored the changes of neuronal and astrocyte cells in pups after hypoxia-ischemia stimulation. Our results indicate that hypoxia-ischemia leads to massive death of neuronal cells and astrocyte proliferation. We also found that in the HIE models, GSPE pretreatment protects neuronal cells and inhibits astrocyte overexpression. Therefore, we initially speculated that the neuroprotective mechanism of GSPE involved in HIE pups may be related to the expression changes of these two cell populations.

\section{Discussion}

HIE involves neonatal suffocation during the perinatal period and cerebral blood perfusion reduces brain damage [14]. The term infant morbidity rate is approximately $1 / 1000$ to $3 / 1000$, and the mortality rate is $15 \%-20 \%$; approximately $25 \%-30 \%$ of survivors have serious sequelae [15]. At present, our understanding of the specific mechanism and pathologic changes in HIE are limited. The mainstream view is that the pathogenesis of HIE is mainly the result of cross-effects, such as autophagy, apoptosis, inflammation, and oxidative stress. In this study we observed the expression of NeuN and GFAP protein in a murine HIE model. The results of immunofluorescence and Western blot demonstrated that GSPE pre-treatment protected neuronal cells, inhibited hyperplasia of astrocytes, and prevented hypoxia/ ischemic brain damage

The role of astrocytes in central nervous system diseases remains controversial. It is well-known that in normal brain tissue astrocytes play an important positive role in guiding the movement of neurons, providing nutrients to neurons, and maintaining the ion balance around neurons. Some scholars believe that astrocytes can participate in the inflammatory response by releasing various cytokines, such as IL-1 $\beta$ and
TNF- $\alpha$, and activation of astrocytes can increase neurotoxicity [16-17].

In our study, compared with the HI group, the expression of GFAP was significantly reduced after GSPE pre-treatment. We believe that GSPE pre-treatment can significantly inhibit the excessive proliferation of astrocytes. In fact, we observed an accumulation of astrocytes in the ipsilateral brain accompanied with a significant decrease in neuronal cells in the murine HIE model, but also noticed that the brain tissues have a significant damage in the hippocampus. Therefore, whether or not astrocytes play an active neuroprotective role in the post-injury stress response or promote the production of inflammatory cytokines and increase the neurotoxicity of HIE in the HIE pathologic process is controversial.

Based on our current experimental data we conclude that in the HIE model, GSPE pre-treatment protects neuronal cells and inhibits hyperplasia of astrocytes. As a common plant extract, GSPE is likely to be a new promising drug for the effective treatment of HIE.

\section{Acknowledgements}

This project was financially supported by the Natural Science Foundation of Guangdong Province (2018A030313579), the Science Foundation of Guangdong No. 2 Provincial People's Hospital for Youth (grant no. YQ2017-001), the Science and Technology Programs in Educational Commission of Guangdong Province (2016), the Innovative and Efficient Projects of Guangdong Pharmaceutical University (2016), and the Guangdong Province Innovation and Entrepreneurship Training Program for University Students (grant no. 201710573046).

\section{Conflict of Interest Statement}

All the authors do not have any possible conflicts of interest. 


\section{References}

[1] Liu G, Li Z G, Gao J S. Hypothermia in neonatal hypoxic-ischemic encephalopathy (HIE) [J]. Euro Review Medi Pharmacological Sci, 2017, 21: 50-53.

[2] Rao R, Trivedi S, Distler A, et al. Neurodevelopmental Outcomes in Neonates with Mild Hypoxic Ischemic Encephalopathy Treated with Therapeutic Hypothermia [J]. Am J Perinatol, 2019.

[3] Zhou X M, Liu J, Wang Y, et al. Silencing of long noncoding RNA MEG3 enhances cerebral protection of dexmedetomidine against hypoxic-ischemic brain damage in neonatal mice by binding to miR-129-5p [J]. J Cell Biochem, 2018.

[4] Xu B, Xiao A J, Chen W, et al. Neuroprotective effects of a PSD-95 inhibitor in neonatal hypoxic-ischemic brain injury [J]. Molecular Neurobi, 2016, 53: 5962-5970.

[5] Luo Z, Zhang M, Niu X, et al. Inhibition of the PI3K/Akt signaling pathway impedes the restoration of neurological function following hypoxic-ischemic brain damage in a neonatal rabbit model [J]. J Cell Biochem, 2019.

[6] Devi A, Jolitha A B, Ishii N. Grape Seed Proanthocyanidin Extract (GSPE) and antioxidant defense in the brain of adult rats [J]. Medi Sci Mo, 2006, 12: BR124-BR129.

[7] Liu B, Zhang H, Tan X, et al. GSPE reduces lead-induced oxidative stress by activating the Nrf2 pathway and suppressing miR153 and GSK-3 $\beta$ in rat kidney [J]. Oncotarget, 2017, 8: 42226-42237.

[8] Rajput S, Sun L, Zhang N Y, et al. Grape Seed Proanthocyanidin Extract Alleviates AflatoxinB1-Induced Immunotoxicity and Oxidative Stress via Modulation of NF- $\kappa \mathrm{B}$ and Nrf2 Signaling Pathways in Broilers [J]. Toxins, 2019, 11: 23 .
[9] Guo F, Hu Y, Niu Q, et al. Grape Seed Proanthocyanidin Extract Inhibits Human Esophageal Squamous Cancerous Cell Line ECA109 via the NF- $\kappa$ B Signaling Pathway [J]. Mediators Inflamm, 2018, 22: 6999-7012.

[10] Hao J P, Shi H, Zhang J, et al. Role of GSPE in improving early cerebral vascular damage by inhibition of Profilin-1 expression in a ouabain-induced hypertension model [J]. Euro Review Medi Pharmacol Sci, 2018, 22: 6999-7012.

[11] Sanna R S, Muthangi S, BK C S, et al. Grape seed proanthocyanidin extract and insulin prevents cognitive decline in type 1 diabetic rat by impacting Bcl-2 and Bax in the prefrontal cortex [J]. Metab Brain Dis, 2018: 1-15.

[12] Fang M, Jiang H, Ye L, et al. Metformin treatment after the hypoxia-ischemia attenuates brain injury in newborn rats $[\mathrm{J}]$. Oncotarget, 2017, 8: 75308-75325.

[13] Al Mamun $\mathrm{A}, \mathrm{Yu} \mathrm{H}$, Romana $\mathrm{S}$, et al. Inflammatory Responses are Sex Specific in Chronic Hypoxic-Ischemic Encephalopathy [J]. Cell Trans, 2018.

[14] Ye L, Feng Z, Doycheva D, et al. CpG-ODN exerts a neuroprotective effect via the TLR9/pAMPK signaling pathway by activation of autophagy in a neonatal HIE rat model [J]. Experi Neuro, 2018, 301: 70-80.

[15] Jiang W, Guo M, Gong M, et al. Vitamin A bio-modulates apoptosis via the mitochondrial pathway after hypoxic-ischemic brain damage [J]. Mole Brain, 2018, 11: 14.

[16] Birla H, Rai S N, Singh S S, et al. Tinospora cordifolia Suppresses Neuroinflammation in Parkinsonian Mouse Model [J]. NeuroMole Medi, 2019: 1-12.

[17] Teismann P, Schulz J B. Cellular pathology of Parkinson's disease: astrocytes, microglia and inflammation [J]. Cell Tissue Res, 2004, 318: 149-161. 